Pacific Journal of Mathematics

ON THE LEAST PRIMITIVE ROOT OF A 


\section{ON THE LEAST PRIMITIVE ROOT OF A PRIME}

\section{PaUl ERdös and Harold N. Shapiro}

1. Introduction. The problem of estimating the least positive primitive root $g(p)$ of a prime $p$ seems to have been first considered by Vinogradov. His first result was [4, v. 2 part 7 chap. 14]

$$
g(p) \leqq 2^{m} p^{1 / 2} \log p,
$$

where $m$ denotes the number of distinct prime factors of $p-1$. In 1930 , [6], he improved this to

$$
g(p) \leqq 2^{m} \frac{p-1}{\phi(p-1)} p^{1 / 2}
$$

where $\phi(n)$ is the Euler $\phi$-function. Next, in 1942, Hua [3] improved this to

$$
g(p)<2^{m+1} p^{1 / 2},
$$

and obtained also, for the primitive root of least absolute value, $h(p)$,

$$
|h(p)|<2^{m} p^{1 / 2} .
$$

Lastly, Erdös [2] proved that for $p$ sufficiently large

$$
g(p)<p^{1 / 2}(\log p)^{17} .
$$

This last result, of course, is not directly comparable with the others, giving better results for some primes and worse results for others.

In any event, all of the results are very weak (as is evidenced by a glance at tables of primitive roots [1]) in relationship to the conjecture that the true order of $g(p)$ is about $\log p$. In this connection, Pillai [5] has proved

$$
g(p)>\log \log p
$$

for infinitely many $p$.

In this note we shall give a very simple way of handling character sums, which not only yields (1.3) and (1.4) but allows a small improvement of these results; for example

$$
g(p)=O\left(m^{c} p^{1 / 2}\right), \quad(c \text { a constant }) .
$$

Received March 12, 1956. 
2. A lemma concerning character sums. We consider first an inequality for certain character sums on which our later estimates will depend. Let $S$ and $T$ be any two sets of integers, such that modulo a given prime $p$, no two integers of $S$ are congruent, and no two integers of $T$ are congruent. Denote by $N(S), N(T)$ the number of integers in $S$ and $T$ respectively. We have

Lemma. For $\chi$ a non-principal character modulo $p$,

$$
\left|\sum_{\substack{u \in S \\ v \in T}} \chi(u+v)\right| \leqq p^{1 / 2} \sqrt{N(S) N(T)}
$$

Proof. Set

$$
\tau(\chi)=\sum_{h=1}^{p} \chi(h) e^{2 \pi i h / p} .
$$

It is well known that $|\tau(\chi)|=p^{1 / 2}$, for $\chi$ a non-principal character. Also,

$$
\tau(\bar{\chi}) \chi(t)=\sum_{h=1}^{p} \bar{\chi}(h) e^{2 \pi i h t / p} .
$$

From this we get

$$
\tau(\bar{\chi}) \sum_{\substack{u \in S \\ v \in T}} \chi(u+v)=\sum_{\substack{u \in S \\ v \in T}} \sum_{h=1}^{p} \bar{\chi}(h) e^{2 \pi i n / p \cdot(u+v)} .
$$

Then taking absolute values and using Schwarz's inequality

$$
\begin{aligned}
p^{1 / 2}\left|\sum_{\substack{x \in S \\
v \in T}} \chi(u+v)\right| & \leqq \sum_{h=1}^{p}\left|\sum_{u \in S} e^{2 \pi i h u / p}\right|\left|\sum_{v \in T} e^{2 \pi i h v / p}\right| \\
& \leqq\left\{\sum_{h=1}^{p}\left|\sum_{u \in S} e^{2 \pi i h u / p}\right|^{2} \sum_{h=1}^{p}\left|\sum_{v \in T} e^{2 \pi i h v / p}\right|^{2}\right\}^{1 / 2} .
\end{aligned}
$$

But

$$
\begin{aligned}
\sum_{h=1}^{p}\left|\sum_{u \in S} e^{2 \pi i h u / p}\right|^{2} & =\sum_{h=1}^{p} \sum_{\substack{u_{1} \in S \\
u_{2} \in S}} e^{2 \pi i h / p \cdot\left(u_{1}-u_{2}\right)} \\
& =\sum_{\substack{u_{1} \in S \\
u_{2} \in S}} \sum_{h=1}^{p} e^{2 \pi i h / p \cdot\left(u_{1}-u_{2}\right)}=p N(S) .
\end{aligned}
$$

Similarly

$$
\sum_{h=1}^{p}\left|\sum_{v \in T} e^{2 \pi i \hbar v / p}\right|^{2}=p N(T)
$$


and the lemma follows immediately.

3. Another proof of Hua's result. By way of illustrating the manner in which the above lemma is to be applied we give here another proof of (1.3). It is well known that if $t$ is not a primitive root modulo $p$ then

$$
P(t)=\sum_{d \mid p-1} \frac{\mu(d)}{\phi(d)} \sum_{\alpha(x)=d} \chi(t)=0
$$

where $o(\chi)=d$ denotes that the inner summation is taken over all characters of order $d$.

Now if $x+1=g(p)$, the smallest positive primitive root mod $p$, we see that $P(t)=0,1 \leqq t \leqq x$. Thus let $S=T$ denote the set of integers $1,2, \cdots,[x / 2]$; we have

$$
\begin{aligned}
& 0=\sum_{d \backslash p-1} \frac{\mu(d)}{\phi(d)} \sum_{o(x)=d} \sum_{\substack{u \in S \\
v \in T}} \chi(u+v) \\
& =[x / 2]^{2}+\sum_{\substack{d \mid p-1 \\
d>1}} \frac{\mu(d)}{\phi(d)} \sum_{o(x)=a} \sum_{\substack{u \in S \\
v \in T}} \chi(u+v) .
\end{aligned}
$$

Applying the lemma, this gives

$$
\left(2^{m}-1\right) p^{1 / 2}[x / 2] \geqq[x / 2]^{2}
$$

or

$$
[x / 2] \leqq\left(2^{m}-1\right) p^{1 / 2} .
$$

Since $2[x / 2]+2 \geq x+1=g(p)$ this yields

$$
g(p) \leqq 2^{m+1} p^{1 / 2}-2 p^{1 / 2}+2<2^{m+1} p^{1 / 2}
$$

which is Hua's result (1.3).

Similarly, if in the above argument we use for $S=T$ the set of nonzero integers $-[x / 2], \cdots,[x / 2]$ where $x+1=|h(p)|$, we are led immediately to the result (1.4).

4. A small improvement in the estimate. The facility with which the lemma of $\S 2$ enables us to handle the relevant character sums makes possible an improvement of the estimates for $g(p)$ and $h(p)$. We consider only the case of $g(p)$, since a similar estimate for $h(p)$ then follows automatically.

Let $F_{x}(d)$ denote the number of integers among

$$
u+v, 1 \leqq u \leqq[x / 2], 1 \leqq v \leqq[x / 2]
$$


such that $u+v$ is a $d$ th power residue modulo $p$. Then, letting $S$ denote the set of integers $1,2, \cdots,[x / 2]$, we have

$$
\begin{aligned}
F_{x}(d) & =\frac{1}{d} \sum_{\substack{u \in S \\
v \in S}} \sum_{o(x) \mid d} \chi(u+v) \\
& =\frac{1}{d}[x / 2]^{2}+\frac{1}{d} \sum_{\substack{o(x) \mid a \\
o(x)>1}} \sum_{\substack{u \in S \\
v \in S}} \chi(u+v) .
\end{aligned}
$$

Applying the lemma of $\S 2$ we obtain

$$
F_{x}(d)=\frac{x^{2}}{4 d}+O\left(x p^{1 / 2}\right) .
$$

If we let $N(x)$ denote the numbers among the

$$
u+v, \quad u \in S, \quad v \in S
$$

which are primitive roots modulo $p$, it is easily seen that

$$
N(x)=\sum_{d \mid p-1} \mu(d) F_{x}(d) .
$$

Applying Brun's method to (4.2), in conjunction with (4.1), in order to make a lower estimate for $N(x)$, one obtains

$$
N(x)>\frac{x^{2}}{4} \sum_{a \mid p-1} \frac{\mu(d)}{d}+O\left(m^{c} p^{1 / 2} x\right)
$$

or

$$
N(x)>\frac{\phi(p-1)}{p-1} \frac{x^{2}}{4}+O\left(m^{c} p^{1 / 2} x\right)
$$

Thus if we take $x+1=g(p), N(x)=0$ and (4.3) yields

$$
x=O\left(\frac{p-1}{\phi(p-1)} m^{\mathrm{c}} p^{1 / 2}\right) .
$$

Finally since

$$
\frac{p-1}{\phi(p-1)}=\prod_{q \mid p-1} \frac{1}{1-1 / q}<\prod_{i=1}^{m} \frac{1}{1-1 / p_{i}}=O(\log m)=O\left(m^{\varepsilon}\right)
$$

(where $p_{i}$ denotes the $i$ th prime), (4.4) gives

$$
x=O\left(m^{c} p^{1 / 2}\right),
$$

and hence

$$
g(p)=O\left(m^{c} p^{1 / 2}\right)
$$


which is the desired result.

\section{REFERENCES}

1. Allan Cunningham, H. J. Woodall and T.G. Creak, On least primitive roots, Proc. London Math. Soc. 2nd series, 21 (1922-23), 343-358.

2. P. Erdös, Least primitive root of a primc, Bull. Amer. Math. Soc., 55 (1945), 131132.

3. L. K. Hua, On the least primitive root of prime, Bull. Amer. Math. Soc., 48 (1942), 726-730.

4. E. Landau, Vorlesungen über Zahlentheorie, Leipzig 1927, (New York 1947).

5. S. Pillai, On the smallest primitive root of a prime, J. Indian Math. Soc., 8 (1944), 14-17.

6. I. M. Vinogradov, On the least primitive root of a prime, Dokl. Akad. Nauk, S.S.S.R. (1930), 7-11.

NOTRE DAME UNIVERSITY

NEW YORK UNIVERSITY 



\section{PACIFIC JOURNAL OF MATHEMATICS}

EDITORS

H. L. ROYDEN

Stanford University

Stanford, California

R. A. Beaumont

University of Washington

Seattle 5 , Washington
A. R. Whiteman

University of Southern California

Los Angeles 7, California

E. G. Straus

University of California

Los Angeles 24, California

\section{ASSOCIATE EDITORS}
E. F. BECKENBACH
C. E. BURGESS
M. HALL
E. HEWITT
A. HORN
V. GANAPATHY IYER
R. D. JAMES
M. S. KNEBELMAN

L. NACHBIN

I. NIVEN

G. SZEKERES

T. G. OSTROM

M. M. SCHIFFER
F. WOLF

K. YOSIDA

\section{SUPPORTING INSTITUTIONS}

UNIVERSITY OF BRITISH COLUMBIA

CALIFORNIA INSTITUTE OF TECHNOLOGY

UNIVERSITY OF CALIFORNIA

MONTANA STATE UNIVERSITY

UNIVERSITY OF NEVADA

OREGON STATE COLLEGE

UNIVERSITY OF OREGON

UNIVERSITY OF SOUTHERN CALIFORNIA

\author{
STANFORD UNIVERSITY \\ UNIVERSITY OF UTAH \\ WASHINGTON STATE COLLEGE \\ UNIVERSITY OF WASHINGTON \\ AMERICAN MATHEMATICAL SOCIETY \\ CALIFORNIA RESEARCH CORPORATION \\ HUGHES AIRCRAFT COMPANY \\ THE RAMO-WOOLDRIDGE CORPORATION
}




\section{Pacific Journal of Mathematics}

\section{Vol. 7, No. $1 \quad$ January, 1957}

Richard Davis Anderson, Zero-dimensional compact groups of

homeomorphisms ................................... 797

Hans-Joachim Bremermann, Holomorphic functionals and complex

convexity in Banach spaces........................... 811

Hugh D. Brunk, G. M. Ewing and W. R. Utz, Minimizing integrals in

certain classes of monotone functions ................. 833

Philip David, Uniqueness theory for asymptotic expansions in general

regions ...................................... 849

Paul Erdős and Harold Nathaniel Shapiro, On the least primitive root of a

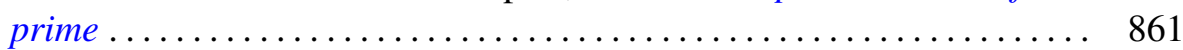

Watson Bryan Fulks, Regular regions for the heat equation ........... 867

William Robert Gaffey, A real inversion formula for a class of bilateral

Laplace transforms ................................ 879

Ronald Kay Getoor, On characteristic functions of Banach space valued random variables ................................. 885

Louis Guttman, Some inequalities between latent roots and minimax (maximin) elements of real matrices ...................... 897

Frank Harary, The number of dissimilar supergraphs of a linear graph .... 903

Edwin Hewitt and Herbert S. Zuckerman, Structure theory for a class of convolution algebras .................................. 913

Amnon Jakimovski, Some Tauberian theorems . . . . . . . . . . . . . . . . . 943

C. T. Rajagopal, Simplified proofs of "Some Tauberian theorems" of Jakimovski................................

Paul Joseph Kelly, A congruence theorem for trees ................. 961

Robert Forbes McNaughton, Jr., On the measure of normal formulas...... 969

Richard Scott Pierce, Distributivity in Boolean algebras .............. 983

Calvin R. Putnam, Continuous spectra and unitary equivalence ......... 993

Marvin Rosenblum, Perturbation of the continuous spectrum and unitary

equivalence................................... 997

V. N. Singh, Certain generalized hypergeometric identities of the

Rogers-Ramanujan type.......................

Peter Swerling, Families of transformations in the function spaces $H^{p} \ldots \ldots 1015$ 